\title{
Difficult Patient Behavior in Dialysis Facilities
}

\author{
Danielle Janosevic Aileen X. Wang Jay B. Wish \\ Division of Nephrology, Indiana University, Indianapolis, IN, USA
}

\author{
Keywords \\ Behavior · Dialysis · Nonadherence · Conflict · Involuntary \\ discharge
}

\begin{abstract}
Difficult behavior exhibited by dialysis patients is a spectrum that includes nonadherence, verbal or physical abuse, and threatening acts. Such behaviors may lead to harmful consequences to the patient, other patients, the facility, and staff and can culminate in involuntary discharge. It is important to recognize that these "difficult behaviors" may be due to underlying psychosocial or medical issues, which places an onus on care providers to explore further. According to the Conditions for Coverage $(\mathrm{CfC})$ for dialysis facilities, it falls upon the medical director to coordinate and oversee policies for patient satisfaction, patient safety and rights, involuntary discharges, and adverse events and outcomes. Thus, medical directors are liable for their own actions, and their staff for which they have oversight, for harm or perceived harm to patients in response to difficult behaviors. Guidelines to deal with specific patient behavior scenarios have been published by the Decreasing Dialysis Patient Conflict National Task Force of the Forum of end-stage renal disease (ESRD) Networks. The common denominator for these diffi-
\end{abstract}

cult scenarios is impaired communication, and the majority of patient concerns involve issues with staff, policies, treatments, and diet. Involuntary discharge of a patient should always be viewed as a last resort, and there is a structured process described in the $\mathrm{CfC}$ that requires the involvement of the respective ESRD Network and the facility medical director. As physicians, we are bound by ethical and growing legal obligations to act in an appropriate, ethical, and fair manner to patients who are considered to be "difficult."

(c) 2018 S. Karger AG, Basel

\section{Introduction}

Difficult patient behavior is a spectrum of apparent behavioral or physical aberrancies that pose a perceived risk to the patient, other individuals, or the facility [1-3]. This ranges from dialysis treatment or medication nonadherence to threatening verbal or physical abuse. Due to an increased incidence of difficult patient behavior over recent years [4], these behaviors have spurred studies, debate, and publications in the fields of ethics and law regarding the role of caregivers. The challenges dialysis patients face on a day-to-day basis include significant symptom burden, strict adherence to fluid and dietary intake,

\section{KARGER}

(c) 2018 S. Karger AG, Basel

E-Mail karger@karger.com

www.karger.com/bpu
Jay B. Wish, MD

Division of Nephrology, IU Health University Hospital

550 N. University Blvd., Suite 6100

Indianapolis, IN 46202 (USA)

E-Mail jaywish@earthlink.net 
time constraints to dialysis treatment sessions, and a significant pill burden, all of which lead to patient-perceived poor quality of life [5]. It is important to maintain objectivity and to recognize that the difficult behavior or situation is not a characteristic of the patient, but of a specific set of circumstances that may cause these behaviors $[2,4,6]$.

\section{Specific Examples of Difficult Behaviors}

Nonadherence is an example of a difficult behavior, which has a significant negative effect on patient outcomes. The American Kidney Fund (AKF) conducted a survey [7] to explore difficult behaviors in dialysis patients. The survey consisted of 3,001 randomly selected dialysis patients from the US AKF grant funding database. There was a patient response rate of $40 \%$. Renal physicians were emailed the survey with a response rate of $2.6 \%$. Discrete categories of difficult behaviors were categorized as: missing dialysis/terminating treatment early, medication nonadherence, nonadherence to a prescribed diet or fluid restrictions, or being sedentary. Results of the survey demonstrated that $36 \%$ of patients sign off of dialysis treatments early, $18 \%$ of patients skip treatments, $23 \%$ do not adhere to medication, and over $50 \%$ of patients do not adhere to dietary or fluid intake recommendations. Factors cited in this survey for the nonadherent behaviors were lack of perceived benefit, feeling unwell, forgetfulness, anxiety, depression, and a prominent lack of communication between the physician and patient [7]. Furthermore, arriving late to appointments or refusing procedures such as needle placement also delays treatments and results in further disruption of scheduling and patient care [2].

Physical and verbal abuse by the patient toward dialysis staff or others is the most extreme example of difficult patient behavior and may result in involuntary discharge from the dialysis facility. Examples include bringing firearms to appointments, threatening harm to self or others, verbally abusing staff/other patients, property damage, and dismissiveness of payment obligations [2].

\section{What Causes Difficult Behaviors?}

Prior reviews and meta-analyses $[4,6,8]$ have revealed that anxiety, depression, and cognitive impairment of dialysis patients may contribute toward disrup- tive behaviors. Furthermore, socioeconomic challenges ranging from transportation or lack of a stable home and/or income, education level, and lack of or toxic social support system also tie into behavioral challenges in dialysis patients. In the aforementioned AKF survey, a significant finding was that many of the behaviors across all age groups were attributable mainly to depression, which was present in over $40 \%$ of surveyed patients [7].

The role of depression as a highly significant contributor to nonadherence in dialysis patients has been explored in numerous publications [4, 6, 8-10]. A meta-analysis of available literature examining factors relating to dialytic therapy adherence revealed that depression (either subjective via questionnaire or a DSM4 diagnosis of depression/dysthymia) in this population ranges from 25 to $80 \%$, further confirming the high prevalence of this disorder in end-stage renal disease (ESRD) patients $[4,6]$. Even more significantly, a greater number of dialysis patients are affected by clinical depression $(25-30 \%)$ than patients with CKD not on dialysis (20-25\%), with initiation of dialysis itself associated with increased incidence of depressive symptoms $[4,6,7,10]$.

A commonly cited reason for nonadherence to diet is difficulty following a diet when eating out. In particular, noncompliance with fluid restriction (defined as significant interdialytic weight gain) is especially common in about $25-50 \%$ of dialysis patients [7]. One study hypothesized a possible psychological basis for fluid noncompliance, as these patients would often continue excessive fluid intake despite regular admonishments from their nephrologists about the consequences. The study authors found that circumstances around dialysis sessions can actually lead to conditioning of this behavior. Patients would complain of feeling dizzy and weak after their dialysis session and noted that drinking fluid restored a sense of well-being [11].

The prevalence of anxiety in ESRD populations ranges from 12 to $52 \%$ [10], demonstrating that "nonadherent" behaviors may be an undertreated or undiagnosed panic disorder or generalized anxiety disorder and are frequently associated with substance abuse disorders [10].

Depression is highly associated with cognitive impairment leading to forgetfulness, which was cited as the main reason for medication nonadherence, ignoring dietary restrictions, and noncompliance with dialysis treatment [7]. A study was done in a single dialysis center in South Korea between July and October 2009 among patients aged 20-80 on chronic dialysis for at least 3 months, with- 
out history of diagnosed dementia or depression prior to the study, and not taking drugs that affect cognitive function. The study used a Korean version of the Mini-Mental State Examination to identify patients at risk for depression, and found that $56 \%$ of participants were shown to have depression. Patients with greater depression burden did worse on cognition tests [12].

The AKF survey cited illness/conflict with appointments, lack of communication by healthcare providers as perceived by patients, lack of education materials as cited by healthcare providers, and lack of or toxic social support system as additional reasons for dialysis treatment nonadherence [7]. Stress in various forms - psychosocial, financial, and physical - is also cited as causes of nonadherence [4].

Ghimire et al. [13] confirm several of the above factors as being predictors of medication nonadherence. Their meta-analysis included 44 studies performed over 30 years and found that medication nonadherence rates in dialysis patients ranged from 12 to $98.6 \%$. The analysis attributes the wide variation to heterogenous definitions of nonadherence among the studies. Risk factors for nonadherence were increased number of complex medication regimens, psycho-social variables (e.g., depression), lack of perceived benefit, and younger age. These findings are also confirmed in a qualitative study performed on hemodialysis patients in Australia by some of the same authors [14].

\section{Consequences of Difficult Behaviors}

Difficult behaviors pose not only a risk to the patient's well-being but also to other patients' treatment patterns, and they create a hostile environment in which aggressiveness can escalate. Death or significant morbidity can ensue from nonadherence; the care of patients on shifts during and after a confrontation may also be adversely affected. Severe behavioral issues resulting in involuntary discharge or transfer of the patient from the dialysis facility cause loss of continuity of care, significant emergent healthcare burden, and an investigation of the dialysis unit in question for the action [2]. All available measures must be taken to avoid an involuntary discharge or transfer. The Decreasing Patient Provider Conflict (DPPC) manual from the Forum of ESRD Networks reports that a survey of dialysis facilities in 2002 revealed the leading cause of involuntary transfer/discharges to be nonadherence (25\%) with $8 \%$ due to threat by the patient [3] (Table 1 ).
Table 1. Reasons for involuntary discharge/transfer from dialysis facilities in the US, 2002

\begin{tabular}{lc}
\hline Reason for involuntary discharge/transfer & Percent \\
\hline Treatment nonadherence & 25 \\
Verbal threat & 8.5 \\
Lack of payment & 5.2 \\
Combination: verbal/physical threat & 5.2 \\
Verbal abuse & 5 \\
\hline
\end{tabular}

Results of 2002 ESRD Network survey citing reasons for involuntary transfer from US dialysis units. Over 70\% of ESRD facilities were included. There was a total of 285,982 patients included in the analysis and $458(0.2 \%)$ were reported to have been involuntarily discharged [3].

Table 2. Conclusions of the dialysis provider-patient conflict taskforce [7]

Physicians have the right to refuse treatment to a violent/abusive patient

Care contracts to guide appropriate behavior may be constructed to avoid future conflicts

A physician may terminate a patient-physician relationship only after documented conflict resolution has been attempted

The dialysis unit staff must make a reasonable attempt if the patient is terminated to seek other dialysis care for the patient

Termination of a patient from a physician/group or facility on grounds of nonadherence alone is not acceptable and conflict resolution must be attempted in this circumstance

\section{Medicolegal Implications of Difficult Behaviors}

The DPPC manual delineates a series of medicolegal responsibilities in the dialysis patient-provider relationship including the entitlement of dialysis providers to payment by patients for treatments [15]. Patients can expect care for their dialysis-related condition from the physician within reasonable limits. Responsibilities/grievances that pose potential violation of the physician-patient relationship are classified as harm to self, the facility, or others [1, $2,16]$. On these grounds, the dialysis unit as a whole should follow the guidelines posed by the DPPC shown in Table 2. The effect of noncompliance is grave, with an increasing burden on emergent medical care for missed dialysis, exacerbation of comorbidities, and increased mortality.

While it is clear that difficult patient behaviors impede various aspects of care delivery, the effect of such behav- 
iors is also subject to litigation in categories delineating autonomy and right to refusal of dialysis $[17,18]$ and negligence or abandonment in the case of involuntary discharge or not dialyzing a patient due to a variety of behavioral issues [19]. In a landmark case, Brown versus Bower, it was determined that private dialysis facilities have the right to discharge noncompliant patients $[1,19]$. In this case, a patient (Brown) sued Dr. Bower, his practicing nephrologist, for allegedly violating several federal statues when Dr. Bower refused to take Mr. Brown back as a patient under his care after discharging Mr. Brown due to various threats [19]. It was determined that although Dr. Bower himself did not have to resume care for Mr. Brown, the hospital in which Dr. Bower worked was required to render dialysis care due to the fact that the hospital receives Hill Burton funding [19]. Under this circumstance, the physician's care was rendered under condition of a patient care contract, which can be terminated by either party [19]. However, emergent dialysis by the hospital cannot be withheld if there is no other reasonably available option [1]. Keeping this in mind, it is important for the dialysis unit staff to maintain a paper trail in the event that an involuntary discharge becomes necessary. The nature of this documentation is clearly defined for reference in the DPPC manual (see the Section "Dialysis Unit Staff Management of Difficult Behaviors") [3].

\section{Dialysis Unit Staff Management of Difficult Behaviors}

The shift toward centering decisions around patient experiences, while respecting autonomy and ensuring that decisions do no further harm [5], is paramount in addressing difficult behaviors. Once a difficult behavior is identified as nonadherence, facility disruption, failure to pay, or physical or verbal harm/abuse, the behavior should then be risk classified. There are 3 categories of risk delineated by DPPC manual and cited by others: [2, $3,16]$ risk to self, facility, or others. Once categorized, an attempt to rule out underlying circumstances for the behavior should be investigated. Jones and Goldman [2] have suggested a useful algorithm to approach the difficult dialysis patient in which a problematic behavior is identified, to objectively identify the issue at hand, and investigated in an unbiased manner while considering psychological, metabolic, and environmental stressors. A team is convened to address and propose solutions for the conflict and, if it cannot be resolved, the involvement of the ESRD Network is recommended. In this scenario, all attempts are made to resolve the conflict prior to considering involuntary discharge of the patient. The DPPC manual provides a useful conflict resolution strategy, which is supported by the mnemonic "CONFLICT": Create a calm environment, Open yourself to understanding others, Need a nonjudgmental approach, Focus on the issue, Look for solutions, Implement agreement, Continue to communicate, Take another look [3]. The mnemonic is an easy-to-remember step-by-step method to deescalate distressing situations, which emphasizes the need to remain calm and objective and blends problem solving as a patient-provider communicative approach [3]. Attempts at conflict resolution should be addressed in meetings with all involved dialysis unit staff and the patient, and the discussion should be carefully documented. A time-limited trial to achieve conflict resolution should then be implemented with goals for both the patient and the involved dialysis unit staff clearly articulated with timeframes, similar to rapid-cycle continuous quality improvement. If the dialysis unit staff achieves its goals but the patient does not at the end of the time-limited trial (typically 30 days), then consideration can be given to alternative measures $[2,3]$. This approach of a time-limited trial of dialysis can be a good option for patients with uncertain prognosis for depression or other psychiatric disorders. The trial can allow patients to evaluate dialysis's effect on their life and also allows the nephrologist to evaluate patients' response to the treatment. An analogous approach is to incrementally increase dialysis prescription over time for patients with residual renal function that can lessen the effects of dialysis treatment on depressive symptoms due to decreased treatment burden [20]. It is also important to keep in mind that nonadherence and argumentativeness alone are never grounds for involuntary discharge.

Other useful staff resources to consider involve addressing psychological stressors, which are prevalent in the dialysis patient population $[2,4,6,8-10,12,21]$. Interventions centered on practices established in cognitive behavioral therapy and behavioral modification (such as relaxation and imagery) have been effective in improving adherence to dialysis sessions, dietary compliance, and depression/anxiety symptoms [21].

\section{Conclusions}

Managing provider-patient conflict is an increasingly important topic related to dialysis facilities. A growing ESRD population of diverse socioeconomic back- 
grounds provides an expanding set of circumstances from which the nephrology professional must objectively identify and understand "difficult behaviors." Depression, anxiety, mental illness, and perceived poor quality of life in an ever-expanding diverse dialysis patient population, make it imperative to maintain objectivity when treating our patients. As healthcare providers, we must understand that difficult behavior is usually the result of a set of circumstances, illness, untreated mental health issues, or logistical barriers rather than the patient being a "bad person." Other than violence, involuntary discharge is the most undesirable outcome of "difficult behavior" and can often be avoided by employing successful conflict resolution strategies such as is embodied in the "CONFLICT" mnemonic [3]. The DPPC manual from the Forum of ESRD Networks [3] has clearly de- lineated, based on decades of literature and observations, steps toward conflict resolution, which can help prevent involuntary discharges, improve patient adherence, improve both patient and dialysis staff behavior, lead to better patient outcomes, and should be frequently referred to in conflict resolution. A steering committee, led by the physician and empowered by the dialysis unit professional staff, can work in conjunction with patients to create a better environment for patients from all walks of life.

\section{Disclosure Statement}

The authors declare that there are no conflicts of interest to disclose.

\section{References}

1 Friedman EA: Must we treat noncompliant ESRD patients? Semin Dial 2001;14:23-27.

2 Jones ER, Goldman RS: Managing disruptive behavior by patients and physicians: a responsibility of the dialysis facility Medical Director. J Am Soc Nephrol 2015;10:14701475.

3 Forum of ESRD Networks: Decreasing Dialysis Patient Provider Conflict. http://esrdnetworks.org/resources/special-projects/copy_ of_DPPCProviderManual.pdf (accessed August 28, 2018).

4 Garcia-Llana H, Remor E, Del Peso G, Selgas $\mathrm{R}$ : The role of depression, anxiety, stress and adherence to treatment in dialysis patients health-related quality of life: a systematic review of the literature. Nefrologia 2014;34: 637-657.

5 Hemmett J, McIntyre CW: A dialysis patient's Choice and a Nephrologist's obligation: the need to understand and value the patient's perspective. Semin Dial 2017;30:3-5.

6 Alosaimi FD, Asiri M, Alsuwayt S, Alotaibi T, Bin Mugren M, Almufarrih A, Almodameg S: Psychosocial predictors of nonadherence to medical management among patients on maintenance dialysis. Int J Nephrol Renovasc Dis 2016;9:263-272.
7 American Kidney Fund: Barriers to Treatment Adherence for Dialysis Patients American Kidney Fund and Adherence Survey, 2018. http://www.kidneyfund.org/assets/ pdf/akf-adherence-report.pdf (accessed August 28, 2018).

8 Tohme F, Mor MK, Pena-Polanco J, Green JA, Fine MJ, Palevsky PM, Weisbord SD: Predictors and outcomes of non-adherence in patients receiving maintenance hemodialysis. Int Urol Nephrol 2017;49:1471-1479.

9 Chilcot J, Wellsted D, Da Silva-Gane M, Farrington K: Depression on dialysis. Nephron Clin Pract 2008;108:c256-c264.

10 Cohen SD, Cukor D, Kimmel PL: Anxiety in patients treated with hemodialysis. Clin J Am Soc Nephrol 2016;11:2250-2255.

11 Streltzer J, Hassell LH: Noncompliant hemodialysis patients. A biopsychosocial approach. Gen Hosp Psychiatry 1988;10:255-259.

12 Jung S, Lee YK, Choi SR, Hwang SH, Noh JW: Relationship between cognitive impairment and depression in dialysis patients. Yonsei Med J 2013;54:1447-1453.

13 Ghimire S, Castelino RL, Lioufas NM, Peterson GM, Zaidi ST: Nonadherence to medication therapy in haemodialysis patients: a systematic review. PLoS One 2015;10:e144119.
14 Ghimire S, Castelino RL, Jose MD, Zaidi STR Medication adherence perspectives in haemodialysis patients: a qualitative study. BMC Nephrol 2017;18:167.

15 Wish JB: What is expected of a medical director in the Centers for Medicare and Medicaid Services Conditions of Coverage? Blood Purif 2011;31:61-65.

16 Hashmi A, Moss AH: Treating difficult or disruptive dialysis patients: practical strategies based on ethical principles. Nat Clin Prac Nephrol 2008;4:515-520.

17 Commissioner of Correction v. Kenneth Myers. 13 Nov 1979. North Eastern Rep Second Ser 1979;399:452-459.

18 Vaqar S, Murray B, Panesar M: The medicallegal responsibilities of a dialysis unit medical director. Semin Dial 2014;27:472-476.

19 Ripley EB: Where does the nephrologist stand with a non-compliant, abusive dialysis patient? Internet J Nephrol 2009;5.

20 Cabrera VJ, Hansson J, Kliger AS, Finkelstein FO: Symptom management of the patient with CKD: the role of dialysis. Clin J Am Soc Nephrol 2017;12:687-693.

21 Krespi MR. Psychosocial interventions to improve outcomes among dialysis patients. Semin Dial 2018;31:65-71. 